\title{
Topological spin transport of photons: the optical Magnus Effect and Berry Phase
}

\author{
K.Yu.Bliokh \\ Institute of Radio Astronomy, 4 Krasnoznamyonnaya st., Kharkov, 61002, Ukraine \\ Yu.P.Bliokh \\ Department of Physics, Technion, Haifa, 32000, Israel
}

\begin{abstract}
The paper develops a modified geometrical optics (GO) of smoothly inhomogeneous isotropic medium, which takes into account two topological phenomena: Berry phase and the optical Magnus effect. By using the analogy between a quasi-classical motion of a quantum particle with a spin and GO of an electromagnetic wave in smoothly inhomogeneous media, we have introduced the standard gauge potential associated with the degeneracy in the wave momentum space. This potential corresponds to the Dirac-monopole-like field (Berry curvature), which causes the topological spin (polarization) transport of photons. The deviations of waves of right-hand and left-hand helicity occur in the opposite directions and orthogonally to the principal direction of motion. This produces a spin current directed across the principal motion. The situation is similar to the anomalous Hall effect for electrons. In addition, a simple scheme of the experiment allowing one to observe the topological spin splitting of photons has been suggested.
\end{abstract}

PACS numbers: 41.20.Jb, 42.15.-i, 03.65.Vf, 05.60.-k

The first consistent analysis of geometrical optics (GO) asymptotic approximation for electromagnetic waves in a smoothly inhomogeneous isotropic media was made in a work by Rytov [1]. There he studied the GO in zero approximation in small parameter $k_{0}^{-1}=c / \omega(\omega$ is the wave frequency); also he gave a well-known law of rotation of electromagnetic wave polarization plane, which corresponds to the first approximation in $k_{0}^{-1}$. Geometrical attributes of this law were described further in detail by Vladimirskiy [2]. In the 1980s Berry [3] and his followers showed that geometrical phases are a general fundamental attribute of dynamic systems, and the Rytov Vladimirskiy polarization plane rotation is an example of Berry phase (see [4] and references therein).

Another fundamental phenomenon that is not contained in Rytov's GO was discovered by Zel'dovich et al. in 1990 5]. It was named "the optical Magnus effect" and consists in that waves of right and left circular polarization propagate in smoothly inhomogeneous medium along different trajectories. Experimentally this phenomenon was confirmed in waveguides in the mode limit. The optical Magnus effect required a theoretical justification in the framework of the GO, and phenomenological theory of this phenomenon was proposed in [6], introducing some additional correction terms in GO equations.

At the same time, recently a considerable attention has been focused on a topological transport of quantum particles with a spin (see 7, 8, 9, 10, 11, 12, 13, 14 and references there). This problem is associated with the concepts of the spin splitting and the spin pumping, and various questions of spintronics. The topological spin transport and Berry phase are two manifestations of the common phenomenon, namely, the initiation of a gauge potential (connection) and a curvature in the space where the particle transport occurs [14]. This gauge potential and the curvature affect both the particle's phase and its motion, giving rise to an additional type of the transport. Since Berry phase shows up in much the same way in nonrelativistic quantum particles with a spin and photons (electromagnetic waves) [4], their topological transport properties are bound to be similar. In this case, the Berry phase corresponds to the Rytov law of polarization evolution, while the topological spin transport corresponds to the optical Magnus effect.

Below we construct the modified GO theory, which corresponds exactly to the contemporary ideas of the topological spin transport of particles. In so doing, we use the analogy between the quasi-classical motion of particles with a spin and the wave propagation in the GO approximation. Within the limits of this correspondence, we should replace the Planck constant $\hbar$ with the GO parameter $k_{0}^{-1}$, while discrete spin projections correspond to discrete values of helicity, which can take the values \pm for photons 15 . The latter corresponds to right-hand and left-hand circular polarizations of waves. (It is precisely the circularly polarized waves that are the eigenmodes in the first approximation of GO 15, 16].) The effects considered (Berry phase and the Magnus optical effect) are automatically accounted for when one introduces the gauge potential that corresponds to the spin 1 and the term degeneracy (the intersection of the dispersion curves) at the origin in the momentum space. It is well known 3, 4, 9, 10, 13] that such gauge potential gives rise to the force field like the Dirac monopole field in the momentum space. In this case, two states of the photon helicity (right-hand and left-hand circular polarizations) correspond to two opposite charges in this field, resulting in splitting of the wave trajectories. The obtained results allow us to conclude that the optical Magnus ef- 
fect is a manifestation of the topological spin transport of photons.

As shown in Berry's pioneer work [3], the gauge potentials and fields appear during the transport of the particle with a spin and are due to the presence of the degeneracy points in the particle's spectrum. In the generic case of the term intersection, the universal expression can be written in adiabatic (quasi-classical) approximation for a field tensor (Berry curvature) $F_{i j}$ :

$$
F_{i j}=m \varepsilon_{i j k} \frac{p_{k}}{p^{3}}, \text { or } \mathbf{F}=m \frac{\mathbf{p}}{p^{3}} .
$$

Here $\mathbf{p}$ stands for a three-dimensional space, in which the transport of the particle is considered (the generic case for the points of degeneracy of Hermitian Hamiltonians corresponds precisely to the three-dimensional space), the degeneracy point is assumed to be in the origin of $\mathbf{p}$-space, $m$ is the projection of the particle's spin (the helicity for relativistic particles), and $\varepsilon_{i j k}$ is the unit antisymmetric tensor. Since the $\mathbf{p}$-space is a three-dimensional one, we write $F_{i j}$ in the form of a pseudovector F, Eq. (11).

Field (curvature) (11) corresponds to a certain gauge potential (connection) $\mathbf{A}$ on the $\mathbf{p}$-space:

$$
\mathbf{F}=\frac{\partial}{\partial \mathbf{p}} \times \mathbf{A}, \quad \text { or } \quad F_{i j}=\frac{\partial A_{j}}{\partial p_{i}}-\frac{\partial A_{i}}{\partial p_{j}} .
$$

The field $\mathbf{F}$ is invariant under gauge transformations of potential, $\mathbf{A} \rightarrow \mathbf{A}+\partial \psi / \partial \mathbf{p}$, and all basic laws for measurable quantities should be expressed in terms of this field. If the coordinate or momentum space acts as the $\mathbf{p}$-space, the potential enters into the Hamiltonian and operates in perfect analogy to an electromagnetic vector-potential in the appropriate space [11, 13]. Thus the perfect analogy is seen between field (11) and the magnetic field of the Dirac monopole in the $\mathbf{p}$-space. Now the effective 'spin charge' $m$ acts as the particle charge.

Let us apply these general statements to a photon or an electromagnetic wave. Taking into account its dispersion $\omega= \pm k c$ (or $E= \pm p c, E$ and $p$ are the photon energy and momentum, respectively), we see that the point $p=$ $k=0$ is the point of the term intersection. Hence the gauge potential $\mathbf{A}$ appears in the momentum space of the photon. This potential corresponds to field (10) with $m= \pm 1$ for right and left circularly polarized photons.

Let us write the Hamiltonian of an electromagnetic wave in GO approximation [17:

$$
H(\mathbf{p}, \mathbf{r})=\frac{1}{2}\left[p^{2}-n^{2}(\mathbf{r})\right]=0 .
$$

Here $n(\mathbf{r})$ is the refractive index of the isotropic smoothly inhomogeneous medium, while $\mathbf{p}=k_{0}^{-1} \mathbf{k}$ is the dimensionless momentum of the wave $(\mathbf{k}$ stands for the wavevector). Note that all calculations are conducted in the first approximation in $k_{0}^{-1}$, that is, up to $O\left(k_{0}^{-2}\right)$.
We must introduce into Hamiltonian (3) the mentioned gauge vector-potential $\mathbf{A}$ operating in the momentum $\mathbf{p}$ space. As a result (compare with 11, 13]) we have for circularly polarized waves:

$$
H(\mathbf{p}, \mathbf{R})=\frac{1}{2}\left[p^{2}-n^{2}\left(\mathbf{R}-k_{0}^{-1} \mathbf{A}(\mathbf{p})\right)\right]=0 .
$$

Here we have switched from the usual coordinates $\mathbf{r}$ to the generalized coordinates $\mathbf{R}$,

$$
\mathbf{r}=\mathbf{R}-k_{0}^{-1} \mathbf{A}
$$

and have accounted for the correspondence $\hbar \leftrightarrow k_{0}^{-1}$ between the quasi-classical quantum mechanics and GO. By expanding Eq.(44) in the Taylor series one can show that it is physically equivalent to the Hamiltonian that has been obtained in [16], and that follows immediately from Maxwell equations. The problem in [16] is considered in fact in the generalized coordinates $\mathbf{R}$, since all properties are derived there from the wave phases, which are determined just by generalized coordinates (see Eq.(91)).

In the generalized coordinates the Hamilton ray equations for Eq.(4) have the canonical form (such equations were studied in 16]). However, the ray equations are more conveniently considered in the ordinary coordinates r, Eq. (515). By using the analogy with a motion of the charged particle in a magnetic field, it is easily understood that the presence of the vector-potential $\mathbf{A}$ in Eq. (4) will produce a force analogous to the Lorentz force with field (11), (2) in a momentum space (see also 7, 11, 13]). As a result we have

$$
\dot{\mathbf{p}}=-\frac{\partial H}{\partial \mathbf{r}}, \quad \dot{\mathbf{r}}=\frac{\partial H}{\partial \mathbf{p}}-k_{0}^{-1}(\mathbf{F} \times \dot{\mathbf{p}}),
$$

where the dot signifies the differentiation with respect to the parameter associated with the ray arc length. By substituting Eq. (10) with $m= \pm 1$ and Eqs. (3)-(15) into Eq.(6), we obtain

$$
\dot{\mathbf{p}}=\frac{1}{2} \frac{\partial n^{2}}{\partial \mathbf{r}}, \quad \dot{\mathbf{r}}=\mathbf{p} \mp k_{0}^{-1}\left(\frac{\mathbf{p}}{p^{3}} \times \dot{\mathbf{p}}\right) .
$$

These are the desired equations, which describe the propagation trajectory of electromagnetic waves (photons) in the first approximation of GO. The first equation in (7) and the second equation without its last term, corresponds to the well-known zero-order approximation of GO (see 17]). The last term in the right-hand side of the second equation in (17) determines the first-order correction in $k_{0}^{-1}$ and is of opposite signs for the waves of right-hand and left-hand circular polarizations. It describes the Magnus optical effect or the topological spin transport of photons. We notice that this term causes an additional displacement of photons of distinct helicity in opposite directions normally to the ray. In other 
words, the spin current of photons across the principal direction of their propagation arises. This situation is in complete agreement with the currently considered topological spin currents of quasi-classical particles 7, 8, 9, 10, 11, 12, 13, 14]. Besides, the term obtained is quite similar to those describing the anomalous Hall effect in a two-dimensional electron gas 8, 9, 10]. This analogy becomes all the more evident if one notes that the anomalous Hall effect is related to the spin-degeneration (in a momentum space) of the terms described by the spin-orbital interaction. At the same time, the Magnus optical effect also was associated with the spin-orbital interaction in [a], and, as we have shown, is caused by the presence of the point of degeneracy in a momentum space. Consequently, the Magnus optical effect may be thought of as a manifestation of the anomalous Hall effect of photons [18].

The topological nature of trajectory splitting (7) implies that it is dictated exclusively by a gauge potential (connection) and a field (curvature), which are connected with the degeneracy in the momentum space. As a consequence, the magnitude of splitting for the rays of left-hand and right-hand polarizations is determined by a contour integral in the momentum space. Indeed, by integrating the trajectory deviations, which are specified by the last term of the second equation in (7), we obtain

$$
\delta \mathbf{r}=\mp k_{0}^{-1} \int_{0}^{s} \frac{(\mathbf{p} \times \dot{\mathbf{p}})}{p^{3}} d s=\mp k_{0}^{-1} \int_{L} \frac{(\mathbf{p} \times d \mathbf{p})}{p^{3}},
$$

where $L$ is the contour in the $\mathbf{p}$-space along which the system moves. Hence the optical Magnus effect is a topological nonlocal effect as the Berry phase is.

The Rytov-Vladimirsky-Berry phase in the theory suggested (Eqs. (4) - (7)) arises in perfect analogy to the Dirac phase of a quantum particle in an electromagnetic potential. Indeed, the wave phase (eikonal) in GO is to be expressed in terms of generalized coordinates, since the action is a function of generalized coordinates. As a result, by constructing the wave phase in terms of the generalized coordinates $\mathbf{R}$ and passing on to the usual coordinates $\mathbf{r}$, we have

$$
\varphi=-\omega t+k_{0} \int_{0}^{s} \mathbf{p} d \mathbf{R}=-\omega t+k_{0} \int_{0}^{s} \mathbf{p} d \mathbf{r}-\int_{0}^{s} \mathbf{A} d \mathbf{p} .
$$

Here we have used Eq.(55) and performed the integration by parts; the term outside the integral is equal to zero because $\mathbf{A p}=0$ for the gauge potential in question. The first two terms and the third term in the right side of Eq. (9) are the ordinary dynamic and geometric phases, respectively. The geometric Berry phase in Eq.(9), being given by a contour integral in the momentum $\mathbf{p}$-space, is equal for closed contours to a flux of the field F, Eq.(1), through the surface spanned on this contour. Clearly this phase is of opposite signs for the waves of righthand and left-hand circular polarizations. In the general case of superposition of two polarizations, the geometric phase leads to the Rytov law about the rotation of the electromagnetic wave polarization plane [4].

From the second equation in (7) and equation (9) the expressions for group and phase velocities of circularly polarized waves follow immediately:

$$
\begin{gathered}
\mathbf{v}_{g} \equiv-\frac{\partial H}{\partial \mathbf{k}} / \frac{\partial H}{\partial \omega}=\frac{c}{n^{2}} \dot{\mathbf{r}}=\frac{c}{n}\left[\mathbf{l} \mp k_{0}^{-1} \frac{(\mathbf{p} \times \dot{\mathbf{p}})}{p^{3}}\right] \\
\mathbf{v}_{p h} \equiv \frac{\partial \varphi / \partial t}{|\partial \varphi / \partial \mathbf{r}|^{2}} \frac{\partial \varphi}{\partial \mathbf{r}}=\frac{c}{n} \mathbf{l}\left[1+k_{0}^{-1} \frac{\mathbf{A} \dot{\mathbf{p}}}{p}\right]
\end{gathered}
$$

Here we have considered the medium as being dispersionfree $(n(\omega)=$ const $)$, took into account that $p=n$ (see Eqs.(3) and (4), and kept in mind that the geometric phase in Eq. (9) is defined over the solutions of zero-order approximation (for details see 16]). It follows from Eqs.(10) and (11) that the spin transport of photons and the optical Magnus effect correspond to the difference in group velocities of the waves, whereas the Berry phase corresponds to the difference in their phase velocities. The group velocities differ mainly in direction (the corrections are directed across the ray), whereas the phase velocities differ in absolute value. The inequality of phase and group wave velocities results in a weak anisotropy of the locally isotropic medium [16]. Evidently the anisotropy results from the initiation of the preferential direction, which is determined by the gradient of the inhomogeneity $\dot{\mathbf{p}} \propto \partial n / \partial \mathbf{r}$ (see Eqs.(7)). Note also that the values of the phase velocities are specified by the gauge potential $\mathbf{A}$ and hence depend on the gauge transformations. This fact does not also lead to contradictions, as the phase velocity here (in contrast to the phase) is not a measurable value.

It should be noted that when substituting $\dot{\mathbf{p}}$ from the first equation in (7) into the second one, we obtain the ray equations in the form suggested by Zel'dovich and Liberman in [6]. Meanwhile, there exist fundamental differences between Ref. [6] theory and the present work along with paper [16]. First of all, attention may be drawn to the difference in the Hamiltonian for the waves in paper [6] and in formula (4) of our work. It is evident that Ref. [6] theory involves many excessive variables, which complicate the problem. In essence, the wave polarization was treated there as an independent dynamical variable with a continuous spectrum of values. At the same time, it is shown in [16] that only the right-hand and left-hand circularly polarized waves are the independent eigen modes in the first approximation of GO. This is consistent with the quantization of photon helicity [15]. As a consequence of this fact, an arbitrary wave of mixed polarization splits into two waves of right-hand and lefthand circular polarizations when propagating through an 


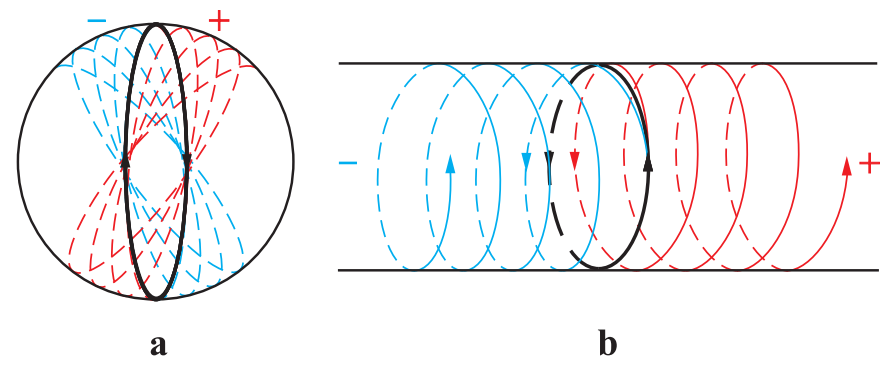

FIG. 1: The trajectories of right-hand (+) and left-hand (-) circularly polarized waves in circular waveguide. Fig.1a shows the shifts of the rays relative to the trajectory of the zero approximation (thick line) in the optical Magnus effect for the meridional ray [5] (view from the waveguide end). Fig.1b shows the splitting of the linearly polarized finite ray into two circularly polarized eigen rays and their subsequent transport along the waveguide in opposite directions (angle view)

inhomogeneous medium. This phenomenon of wave splitting into two independent modes is absent in the paper [6] (for example, according the theory in [6], the trajectory of a linearly polarized wave remains unchanged), but it is in complete agreement with the contemporary conception of the topological spin transport of quantum particles.

Two examples of spin (polarization) photon transport and splitting are presented in Fig.1a and b. There we can see the trajectories of right-hand and left-hand circularly polarized waves in circular waveguides. Fig. 1a is associated with the optical Magnus effect for the meridional ray in a circular waveguide (see [5]), whereas Fig.1b shows the splitting of the linearly polarized finite ray into two rays of right-hand and left-hand polarizations and their subsequent transport along the circular waveguide in opposite directions (see detailed description of these two examples in [16]). The experiment presented in Fig.1b can be performed in a cylindrical structure by exciting an oriented ray associated with the mode of whispering gallery. This experiment will enable one to observe for the first time the spin splitting of photons.

Let us summarize the main outcomes of our work. Exclusively from both the topological considerations and perfect analogy between GO and a quasi-classical motion of quantum particles with a spin, we have constructed the simple and clear scheme of the first GO approximation in $k_{0}^{-1}$ for electromagnetic waves. By introducing in a momentum space the standard gauge potential, which is connected with the point of term degeneracy at the origin, it is possible to describe such phenomena as the optical Magnus effect 5, 6] and the Rytov-Vladimirsky-Berry geometric phase [1, 2, 3, 4]. It becomes apparent that the optical Magnus effect is nothing but the topological spin (polarization) transport of photons, which is similar to the anomalous Hall effect for electrons [8, 9, 10]. It has been shown that the spin photon transport and the optical Magnus effect correspond to the difference in directions of the group velocities of the oppositely polarized waves, whereas the Berry phase is associated with the difference in absolute values of their phase velocities [16]. In the context of the approach suggested the theory is simple and clear and is in perfect agreement with the previous direct derivations [16] following from Maxwell equations. In addition, our theory describes a novel effect, namely, the splitting of a ray of mixed polarization into two rays of right-hand and left-hand circular polarizations. The simple scheme of the experiment for observing this phenomenon has also been suggested (Fig.1b and [16]).

[1] S.M. Rytov, Dokl. Akad. Nauk. SSSR 18, 263 (1938).

[2] V.V. Vladimirskiy, Dokl. Akad. Nauk. SSSR 31, 222 (1941).

[3] M.V. Berry, Proc. R. Soc. A 392, 45 (1984).

[4] A. Shapere and F. Wilczek (ed.), Geometric Phases in Physics (Singapore: World Scientific, 1989); S.I. Vinitskiy, V.L. Debrov, V.M. Dubovik, B.L. Markovski, and Yu.P. Stepanovskiy, Uspekhi Fizicheskih Nauk 160, 6 (1990) [Sov. Phys. Usp. 33, 6 (1990)].

[5] A.V. Dooghin, N.D. Kundikova, V.S. Liberman, and B.Ya. Zel'dovich, Phys. Rev. A, 45, 8204 (1992); B.Ya. Zel'dovich and V.S. Liberman, Kvant. Elektron. 17, 493 (1990) [Sov. J. Quantum Electron. 20, 427 (1990)]; A.V. Dooghin, B.Ya. Zel'dovich, N.D. Kudnikova, and V.S. Liberman, Pis'ma Zh. Eksp. Teor. Fiz. 53, 186 (1991) [Sov. Phys. JETP Letters 53, 197 (1991)]; A.V. Dooghin, B.Ya. Zel'dovich, N.D. Kudnikova, and V.S. Liberman, Zh. Eksp. Teor. Fiz. 100, 1474 (1991) [Sov. Phys. JETP 73, 816 (1991)].

[6] V.S. Liberman and B.Ya. Zel'dovich, Phys. Rev. A 46, 5199 (1992).

[7] G. Sundaram and Q. Niu, Phys. Rev. B 59, 14915 (1999).

[8] T. Jungwirth, Q. Niu, and A.H. MacDonald, Phys. Rev. Lett. 88, 207208 (2002).

[9] D. Culcer, A. MacDonald, and Q. Niu, Phys. Rev. B 68, 045327 (2003).

[10] A.M. Dudarev, R.B. Diener, I. Carusotto, and Q. Niu, cond-mat/0311356r1.

[11] S. Murakami, N. Nagaosa, and S.-C. Zhang, Science 301, 1348 (2003).

[12] B.A. Bernevig, J.P. Hu, E. Mukamel, and S.-C. Zhang, cond-mat/0311024

[13] F. Zhou, cond-mat/0311612

[14] H.-Q. Zhou, S.Y. Cho, and R.H. McKenzie, Phys. Rev. Lett 91, 186803 (2003).

[15] V.B. Berestetskiy, E.M. Lifshits, and L.P.Pitaevskiy Relativistic Quantum Theory, V.1 (Nauka, Moscow, 1968).

[16] K.Yu. Bliokh and Yu.P. Bliokh, physics/0402014

[17] Yu.A. Kravtsov and Yu.I. Orlov, Geometrical Optics of Inhomogeneous Medium (Nauka, Moscow, 1980; Springer-Verlag, Berlin, 1990).

[18] Note, that ordinary Hall effect for photons in magnetic field also was discovered in recent works B.A. van Tiggelen, Phys. Rev. Lett. 75, 422 (1995); G.L.J.A. Rikken and B.A. van Tiggelen, Phys. Rev. Lett. 78, 847 (1997); 
D. Lacoste, F. Donatini, S. Neveu, J.A. Serughetti, and B.A. van Tiggelen, Phys. Rev. E 62, 3934 (2000). 